\title{
A secretion-based dual fluorescence assay for high-throughput screening of alcohol dehydrogenases
}

\author{
Hongyuan $\mathrm{Lu}^{1}$, Shiqin $\mathrm{Yu}^{1}$, Fengyu Qin ${ }^{1}$, Wenbo $\mathrm{Ning}^{1}$, Xiaoqiang $\mathrm{Ma}^{2}$, Kaiyuan Tian ${ }^{1}$, \\ Zhi $\mathrm{Li}^{1}$, and Kang Zhou ${ }^{1}$ \\ ${ }^{1}$ National University of Singapore \\ ${ }^{2}$ Singapore-MIT Alliance for Research and Technology Centre
}

September 2, 2020

\begin{abstract}
Alcohol dehydrogenases (ADHs) play key roles in the production of various chemical precursors that are essential in pharmaceutical and fine chemical industries. To achieve practical application of ADHs in industrial processes, tailoring enzyme properties through rational design or directed evolution is often required. Here, we developed a secretion-based dual fluorescence assay (SDFA) for high-throughput screening of ADHs. In SDFA, an ADH of interest is fused to a mutated superfolder green fluorescence protein (MsfGFP), which could result in secretion of the fusion protein to culture broth. After a simple centrifugation step to remove the cells, the supernatant can be directly used to measure activity of the ADH based on a red fluorescence signal, whose increase is coupled to formation of NADH (a redox co-factor of ADHs) in the reaction. SDFA allows easy quantification of ADH concentration based on the green fluorescence signal of MsfGFP. This feature is useful in determining specific activity and may improve screening accuracy. Out of five ADHs we have tested with SDFA, four ADHs can be secreted and characterized. We successfully screened a combinatorial library of an ADH from Pichia finlandica and identified a variant with a 197-fold higher $\mathrm{kcat} / \mathrm{km}$ value toward (S)-2-octanol compared to its wild-type.
\end{abstract}

\section{Keywords}

Protein secretion, Fluorescence assay, Alcohol dehydrogenase, Directed protein evolution, Cascade reaction

\section{Introduction}

Many drugs and value-added chemicals need to meet stringent enantiopurity standards in pharmaceutical and fine chemical industries. During the last decade, there has been a growing interest in using enzymes for producing chiral chemicals. This is mainly driven by the inherent advantages of biocatalysis over chemical synthesis, such as high chemo-, regio- and enantio-selectivities, mild reaction conditions and using less toxic reagents or solvents (Nealon, Musa, Patel, \& Phillips, 2015; Zheng et al., 2017).

In particular, alcohol dehydrogenases (E.C. 1.1.1.x; $\mathrm{x}=1$ or $2, \mathrm{ADHs}$ ) are among the most investigated redox enzymes. ADHs are a group of $\mathrm{NAD}(\mathrm{P})(\mathrm{H})$-dependent enzymes that catalyze the interconversion between alcohols and ketones or aldehydes. Because of their strict stereoselectivity, ADHs have been employed in the production of various chiral products with high optical purity (Chen, Liu, Lin, \& Zheng, 2016; Kara et al., 2014; Savile, Gruber, Mundorff, Huisman, \& Collier, 2014). However, the performance of wild-type ADHs is rarely adequate for industrial processes. In general, tailoring enzyme properties, such as catalytic activity, 
stereoselectivity, substrate specificity, thermostability and tolerance of organic solvent is often required to realize the practical application of ADHs (R. Zhang, Xu, \& Xiao, 2015).

Directed evolution is one of the most effective methods to engineer ADHs. An ADH may be randomly mutated by using error-prone PCR to identify beneficial mutations. Putative critical residues of an ADH may also be identified based on docking the substrate/product into experimental-determined or predicted tertiary structure of the ADH. Beneficial mutations at these putative residues would be characterized through mutagenesis. Often, multiple beneficial mutations need to be combined and the aforementioned process may be iterated.

In directed evolution, screening the mutants is usually the rate-limiting unit operation, because the rate of hitting a beneficial mutation is low. The assay commonly used to quantify activity of ADH is to monitor the production or depletion of $\mathrm{NAD}(\mathrm{P}) \mathrm{H}$ based on absorbance at $340 \mathrm{~nm}$ as these enzymes couple their enzymatic reactions with the nicotinamide cofactors (Forrest \& Gonzalez, 2000). Usually cells expressing an $\mathrm{ADH}$ was lysed prior to the assay. Unfortunately, this approach is not suitable for high-throughput screening, because of the strong absorption of UV light by cell lysates (Mayer \& Arnold, 2002).

Alternatively, colorimetric assays that combine the redox reaction with a dye-forming reaction have been developed to reduce the noise, because the absorption of visible light by cell lysates was weaker than that of UV light. One of the most widely adopted colorimetric approaches is the nitroblue tetrazolium/phenazine methosulfate (NBT/PMS) assay (Fibla \& Gonzàlez-Duarte, 1993). In the presence of the transferring agent PMS, NBT can be reduced by the $\mathrm{NAD}(\mathrm{P}) \mathrm{H}$ regenerated by an $\mathrm{ADH}$-catalyzed reaction to produce an insoluble blue-purple formazan dye, whose absorbance can be measured at $580 \mathrm{~nm}$. This colorimetric assay has been used in high-throughput screening to identify novel or improved ADHs, such as 6-phosphogluconate dehydrogenase (Huang, Chen, Zhong, Kim, \& Zhang, 2016), glycerol dehydrogenase (H. Zhang, Lountos, Ching, \& Jiang, 2010), halohydrin dehydrogenase (Fox et al., 2007), and phenylacetaldehyde reductase (Makino, Dairi, \& Itoh, 2007). However, this colorimetric assay still needs improvement due to its background noise and limited detection range. Moreover, this assay also fails to enable a continuous measurement of ADHs activity as the final colour product is insoluble, resulting in precipitation that interferes with the absorbance quantification.

In addition, the aforementioned assays generally suffer from two common issues when they are used to screen cells that express enzyme. First, in order to release target enzyme from host cells for determining catalytic activity, cell lysis is often required by these methods. However, once the cells are lysed, together with the enzyme of interest, all cytosolic components are simultaneously released, resulting in a complex matrix that could reduce the sensitivity and accuracy of the assay implemented (Contreras-Llano \& Tan, 2018; Mayer \& Arnold, 2002). For example, cytosolic components, such as nicotinamide cofactor, autofluorescent or chromogenic metabolites may miscount for the catalytic activity of the target enzyme. Non-specific interactions with endogenous enzymes (e.g. endogenous ADHs) could also interfere with the target enzyme reaction if they share similar substrate specificity.

Furthermore, when these assays were applied in high-throughput format, such as microtiter plate screening, the catalytic activity was usually evaluated without normalizing by the amount of enzyme interrogated. Because, it is time-consuming and tedious to quantify the enzyme amount for each variant in a largescale screening effort. Consequently, this inevitably leads to an unfair comparison among the mutants. The quantity of the target enzyme in each mutant sample could vary due to fluctuating protein expression level and nonhomogeneous cell growth rate. Overall, this may not only produce a considerable number of false positives, but also miss out some of the true positives. It will be desirable to have a simple and easy way to quantify the enzyme amount for each mutant so that their catalytic activity can be normalized for a fairer screening.

To tackle these limitations in this study, we developed a secretion-based dual fluorescence assay (SDFA) for high-throughput screening of mutated ADHs. This assay is based on a cascade reaction leading to the formation of a red fluorescent molecule. It also uses a protein secretion system translocating the target 
enzyme to a simpler reaction matrix than cell lysate.

As shown in Figure 1, in the presence of an enzyme (diaphorase), resazurin reacts with the NAD $(\mathrm{P}) \mathrm{H}$ generated from the ADHs-catalysed reaction, leading to the generation of the highly fluorescent moleculeresorufin - whose emission signal can be easily detected at $588 \mathrm{~nm}$ when excited at $535 \mathrm{~nm}$. Since the emission falls within the range of red light, in which fewer Escherichiacoli endogenous molecules emit fluorescence, there is less interference from the reaction mixture and spent culture media.

Meanwhile, we combined the cascade reaction with a protein secretion system which was enabled by a mutated superfolder green fluorescent protein (MsfGFP) (Z. Zhang et al., 2017). By fusing ADHs to MsfGFP, the fusion proteins can be efficiently secreted out of the host cells for enzymatic activity quantification. Therefore, cell lysis was not required by this assay. This led to a much simpler reaction matrix that was free of complex cytosolic components. As a result, the interference caused by intracellular metabolites and endogenous enzymes was eliminated. Also, this secretion system simplified the screening procedure and avoided the potential inconsistency of lysis efficiency.

Additionally, another merit of this assay is that the green fluorescence of the MsfGFP fusions was linearly proportional to its protein amount. This provides a simple and efficient way to quantitate the amount of target enzyme in the reaction matrix. Therefore, normalization of the catalytic activity of different mutants can be easily achieved, further improving the accuracy of the assay.

In this study, we have collected experimental data to support the claimed advantages of SDFA, and successfully demonstrated the usefulness of SDFA through engineering of an ADH, which led to substantial improvement of its catalytic efficiency.

\section{Materials and Methods}

\section{Materials}

All the chemicals and diaphorase used in this study were purchased from Sigma-Aldrich Pte. Ltd., Singapore, unless otherwise stated. The QIAprep Spin Miniprep kit for plasmid extraction was purchased from Qiagen Pte. Ltd., Singapore. The GeneJET gel extraction kit for gel extraction was purchased from Thermo Fisher Scientific, Singapore.

\section{Plasmid and strain construction}

The MsfGFP gene sequence was kindly provided by Dr. Ma Lixin from Hubei University, Wuhan, People's Republic of China. Synthetic ADHs genes and all the used oligonucleotides were ordered from Integrated DNA Technologies. Plasmid construction was carried out based on the Guanine-Thymine standard (Ma et al., 2019). The fusion proteins consist of an N-terminal MsfGFP or eGFP which is separated by a linker with an TEV Protease recognition site sequence (ENLYFQG) from an ADH. The DNA sequence of all the constructed plasmids was verified by Sanger sequencing. A full list of plasmids constructed in this study is provided in Table S1. E coli DH5 $\alpha$ (C2987H, New England Biolabs) was used as cloning host and E. coli BL21 (C2527H, New England Biolabs) was used for protein expression. Plasmid transformation was carried out by using the heat-shock method provided in the manufacturer's instruction.

\section{Protein expression and purification}

A single colony of an E. coli BL21 strain harboring one of the expression plasmids in Table S1 was inoculated into $3 \mathrm{ml}$ of Luria Bertani broth (LB) with $50 \mu \mathrm{g} / \mathrm{ml}$ spectinomycin and grown overnight at $37^{\circ} \mathrm{C} / 250 \mathrm{rpm}$. The overnight-grown culture (using $500 \mu \mathrm{l}$ ) was scaled up with 300 -fold dilution in a 500-ml shake flask and grown to a cell density of 0.6 (optical density [OD600]) at $37^{\circ} \mathrm{C} / 250 \mathrm{rpm}$. Protein expression was induced 
by adding $0.1 \mathrm{mM}$ of isopropyl $\beta$-D-1-thiogalactopyranoside (IPTG) and cells were cultured for 22 hours at $30{ }^{\circ} \mathrm{C} / 250 \mathrm{rpm}$.

For isolation of the intracellularly expressed $(R$ )-2-octanol dehydrogenase (PfODH), cells were harvested by centrifugation at 4,000 g for 10 mins. Cell pellets were then resuspended in equilibrium buffer $(20 \mathrm{mM}$ sodium phosphate, $300 \mathrm{mM}$ sodium chloride, and $10 \mathrm{mM}$ imidazole; $\mathrm{pH}$ 7.4), and mechanically lysed by a cell disrupter (OS, Constant Systems Ltd.). The resulting cell lysate was centrifuged at 10,000 g for 20 mins to obtain the supernatant that contains soluble proteins. For isolation of the extracellularly expressed MsfGFP fusion proteins, the induced cell culture was centrifuged at 4,000 g for 20 mins to obtain the supernatant that accommodates secretory proteins. Target proteins from the above two types of supernatant were both purified by HisPur Ni-NTA Resin (Thermo Fisher Scientific) according to the manufacturer's instruction. Desalting of the protein elution was carried out by using Sephadex G-25 PD-10 columns (GE Healthcare) according to the manufacturer's instruction. The concentration of purified proteins was determined by the Micro BCA Protein Assay kit (Thermo Fisher Scientific). The absorbance of assay mixtures was measured by the Infinite $200^{(\mathrm{r})}$ PRO microplate plate reader (Tecan Group AG). The presence of the purified target proteins was verified by SDS-polyacrylamide gel electrophoresis (SDS-PAGE) using Mini-PROTEAN TGX Precast Protein Gels (4561083, Bio-Rad). Gel image was taken by the Gel Doc EZ Gel Documentation System (Bio-Rad).

\section{Fluorescence observation and SDS-PAGE analysis of supernatant samples}

The protein expression of the MsfGFP/eGFP-ADH fusion proteins was induced as described above. The grown cell cultures were then centrifuged at 4,000 g for 20 mins to obtain the supernatants. To observe the fluorescence of the fusion proteins, the supernatant samples were excited by blue light using a Safe Imager 2.0 Blue Light Transilluminator (Thermo) and the image was taken through an amber filter unit. Afterwards, the supernatant samples were concentrated 5 -fold by Microcon(r) centrifugal filter devices (Merck Millipore) for SDS-PAGE analysis.

\section{The coupled enzyme assay with purified ADH/secreted fusion proteins}

The coupled enzyme assay reagent was prepared as a mixture consisting of $4 \mathrm{U} / \mathrm{ml}$ diaphorase, $1 \mathrm{mM}$ resazurin, $1 \mathrm{mM}$ substrate (for $\mathrm{ADH}$ ), $1 \mathrm{mM} \mathrm{NAD}^{+}$and $0.1 \mathrm{M}$ Tris buffer ( $\mathrm{pH} 8.0$ ). To examine the catalytic activity of PfODH toward various substrates, the purified PfODH was first diluted with 0.1 M Tris buffer to yield a final concentration of $20 \mu \mathrm{g} / \mathrm{ml}$. Subsequently, $50 \mu \mathrm{l}$ of each diluted PfODH sample was then pipetted into the wells of a 96-well back polystyrene microtiter plate (Costar 3603, Corning). Assay reactions were initiated by adding $50 \mu \mathrm{l}$ of the assay mixture to each sample well, immediately followed by a continuous measurement of the fluorescence of resorufin $(\mathrm{Ex} / \mathrm{Em}=535 / 588 \mathrm{~nm})$ with the "Kinetic Measurement" mode in i-control software for 10 mins by the Infinite $200{ }^{\circledR}$ PRO microplate plate reader (Tecan Group AG).

The same procedure was used for determining the catalytic activity of secreted fusion proteins. Cultures of the MsfGFP-fused ADHs were centrifuged at 4,000 g for 20 mins to obtain the supernatant that accommodates secretory proteins. $50 \mu \mathrm{l}$ of the supernatant sample was used for the assay. Similarly, for high throughput screening experiment, induced cell cultures in 96 deep well plates (Greiner Bio-One GmbH) were centrifuged at 4,000 g for 20 mins. $50 \mu \mathrm{l}$ of the supernatant sample from each mutant was transferred to a well in a 96well microtiter plate, and the fluorescence of MsfGFP-fused mutants $(\mathrm{Ex} / \mathrm{Em}=488 / 525 \mathrm{~nm})$ was determined first by plate reader. Subsequently, $50 \mu \mathrm{l}$ of the assay mixture was added to the well, immediately followed by a continuous measurement of the fluorescence of resorufin $(\mathrm{Ex} / \mathrm{Em}=535 / 588 \mathrm{~nm})$ by plate reader.

\section{Kinetic analysis}

Kinetic analysis of the wild-type PfODH and the selected PfODH variant was performed by determining the production of $\mathrm{NADH}(\lambda=340 \mathrm{~nm})$ through absorbance measurement. Assays were carried out in $200 \mu \mathrm{l}$ of 
PBS buffer $(100 \mathrm{mM}, \mathrm{pH} 7.5)$ containing $6 \mathrm{mM} \mathrm{NAD}^{+}$, at room temperature, using $13.3 \mu \mathrm{M}$ of the wild-type $\mathrm{PfODH}$ or $0.055 \mu \mathrm{M}$ of the selected PfODH variant at substrate concentrations ranging from $0.125 \mathrm{mM}$ to $5.0 \mathrm{mM}$. The extinction coefficient used for NADH under the assay conditions was $6220 \mathrm{M}^{-1} \mathrm{~cm}^{-1}$. All assays were performed in triplicate. The enzyme kinetic parameters $\mathrm{k}_{\mathrm{m}}$, and $\mathrm{k}_{\mathrm{cat}}$ were calculated from the enzyme progress curves via the Lineweaver-Burk plot.

\section{Results and discussion}

\section{A cascade reaction enables fluorogenic determination of ADH activity}

As shown in Figure 1, the catalytic activity of ADHs in an oxidation reaction can be expressed as the rate of forming $\mathrm{NAD}(\mathrm{P}) \mathrm{H}$. Although it is possible to quantitate $\mathrm{NAD}(\mathrm{P}) \mathrm{H}$ by directly measuring its light absorbance (UV $340 \mathrm{~nm}$ ), this method suffers from low sensitivity and high background noise (R. Zhang et al., 2015). Alternatively, measuring the fluorescence of $\mathrm{NAD}(\mathrm{P}) \mathrm{H} \mathrm{Ex} / \mathrm{Em}=340 / 445 \mathrm{~nm}$ ) can be used to quantify $\mathrm{NAD}(\mathrm{P}) \mathrm{H}$ and has shown a greater sensitivity compared to the absorbance-based approach (Held, 2007). However, our experiments showed that although the linear correlation between NADH concentration and its fluorescence can be obtained when NADH was serially diluted with deionized water, buffer or fresh media (Figure S1 A-D), such a correlation cannot be established when spent media was used as the diluent (Figure S1 E-F). These results suggest that metabolites released by growing cells can substantially interfere with the fluorescence detection of NADH, and therefore such fluorescence assay is not suitable for determining the NADH level in crude samples such as cell lysate or culture supernatant.

To tackle this issue, we employed an indicator enzymatic reaction in which NADH was used by diaphorase to reduce resazurin into resorufin, which is highly fluorescent (Figure 1). The main advantage of this system is that the long excitation/emission wavelengths of resorufin $(\mathrm{Ex} / \mathrm{Em}=535 / 588 \mathrm{~nm})$ can reduce the interferences from cellular metabolites, because most fluorescent molecules in cell lysates are excited in the UV range (Simeonov \& Davis, 2004). As expected, our results showed that fluorescence of the formed resorufin responded linearly to NADH concentration across different medium backgrounds, including spent media (Figure S1 G-L). Meanwhile, this reaction system offers a much more extended linear detection range (0.1-700 $\mu \mathrm{M})$ compared to NADH fluorescence-based approach (0.1-200 $\mu \mathrm{M}$ when the medium background is deionized water). Additionally, through transforming the unstable NADH into the more stable resorufin, this reaction system provides a more durable and accurate fluorescent signal, allowing a reliable and continuous fluorogenic determination of NADH concentration.

Given these advantages, we may couple an ADH-catalysed reaction with this indicator system to form a coupled enzyme assay for the ADH (Figure 1). To examine whether the ADH activity can be determined by this cascade reaction, we employed an enantioselective $\mathrm{ADH},(R$ )-2-octanol dehydrogenase (PfODH) as the model enzyme. In the cascade reaction mixture, the purified enzyme PfODH was supplied with cofactor $\mathrm{NAD}^{+}$and substrate to initiate target reactions. Concurrently, excessive amount of resazurin and diaphorase were provided in the mixture to stoichiometrically convert NADH into fluorescent resorufin. The enzymatic activity of ADH was thus proportional to the rate at the red fluorescence increased. As shown in Figure 2, the catalytic activity of PfODH toward various substrates, including its native substrate $((R$ )-2-octanol) and other non-native substrates (4-Fluoro- $\alpha$-methylbenzyl alcohol, 1-(3-Methylphenyl)ethanol and 1-Phenylethanol), were determined by following the respective increases in fluorescence of resorufin over time using a plate reader. In contrast, the negative controls without adding PfODH all exhibited no fluorescence increase over time. These results showcased that the coupled enzyme assay can enable a sensitive and continuous, time-dependent monitoring of the ADH-catalysed reaction. 


\section{Incorporating the coupled enzyme assay with a protein secretion system}

High-throughput screening used in enzyme evolution has relied heavily on the use of cell-based approach (Zeymer \& Hilvert, 2018). However, there are two major defects with this method: (i) It requires cell lysis that could introduce interference to the screening assay; (ii) It relies on the false assumption that the amount of enzyme being assayed is uniform across all mutants, therefore resulting in an unfair comparison that consequently increases false positive or negative rates. To further develop our coupled enzyme assay into a better screening method, we set out to address these two limitations.

In particular, we proposed to combine the cascade reaction with a protein secretion system which is based on MsfGFP. The addition of such a secretion system can potentially offer two unique features to our assay. First, it has been reported that when recombinant proteins were fused with MsfGFP, it can lead to the autosecretion of the fusion protein without disturbing their conformation and function (Z. Zhang et al., 2017). Such a non-specific auto-secretion system is highly desirable because cell lysis can therefore be exempted, and the secreted target enzyme can be assayed in the culture broth, which is a much simpler matrix than cell lysate. Second, MsfGFP can serve as a protein expression reporter for its passenger protein. It has been demonstrated that the fluorescence of sfGFP (non-mutated superfolder GFP) fusion protein is linearly proportional to the protein quantity (Pédelacq, Cabantous, Tran, Terwilliger, \& Waldo, 2006). This feature can potentially be used to normalize the enzyme activity of each mutant during high throughput screening, since the fluorescence of MsfGFP fusion protein can be easily and efficiently measured using fluorescence plate reader.

To evaluate whether the MsfGFP-guided secretion system is generally applicable to ADHs, we fused the MsfGFP or eGFP (enhanced GFP, a control which would not lead to protein secretion) to N-terminus of five ADHs, including PfODH from Pichia finlandica, $(S$ )-1,3-butanediol dehydrogenase (CpSADH) from Candida parapsilosis, glycerol dehydrogenase (GlyDH) from E. coli , Meso-2,3-butanediol dehydrogenase (BDH_KP) from Klebsiella pneumoniae and 2,3-butanediol dehydrogenase (BDH_BS) from Bacillus subtilis . The protein expression of the MsfGFP/eGFP-ADH fusion proteins was induced by IPTG. The grown cell cultures were then centrifuged to obtain the supernatants for examining whether the fusion proteins were secreted out of the cells. As shown in Figure 3, all the supernatants of the cells expressing the MsfGFP fusion proteins exhibited fluorescence to various degrees, whereas all the controls did not show any fluorescence. Meanwhile, the presence of secreted MsfGFP fusion proteins and the absence of eGFP fusion proteins in supernatant were further confirmed by SDS-PAGE analysis (Figure 3). Consistent with the fluorescence observation, the respective protein bands of MsfGFP fusion proteins showed varying degrees of staining, further indicating different levels of secretion for the MsfGFP fusion proteins. These results suggest that secretion of five ADHs can be realized by using this MsfGFP-guided auto-secretion system, although with various degree of secretion efficiency.

After we confirmed MsfGFP can enable the secretion of ADHs, we moved forward to examine whether the secreted ADHs' activity can be determined by the coupled enzymatic assay. Assay reaction components were directly added to the culture supernatants of each fusion protein. As shown in Figure 4, the supernatant samples of most MsfGFP-guided ADHs (GlyDH, CpSADH, PfODH, and BDH_KP) generated various fluorescence increases over time through the assay reaction, whereas there was no fluorescence change for all the supernatant sample of eGFP fusion proteins. However, unlike other ADHs, the rate of red fluorescence increase was low for MsfGFP-BDH_BS, which may be mainly due to its low secretion level. Nonetheless, these results demonstrated that the MsfGFP-guided secretion system can be combined with the coupled enzyme assay to establish a secretion-based assay for determining activity of some ADHs.

\section{Normalizing ADH activity by the fluorescence of MsfGFP}

We next assessed the feasibility of using the fluorescence of MsfGFP to normalize the catalytic activity of ADHs among different mutants in high-throughput screening. To mimic the actual screening condition of the secreted ADHs, we purified the fusion protein MsfGFP-PfODH and serially diluted it into different 
concentrations with cell-free spent medium. The green fluorescence $(\mathrm{Ex} / \mathrm{Em}=488 / 525 \mathrm{~nm})$ of the diluted enzyme samples were first determined by fluorescence plate reader. The green fluorescence intensity exhibits a strong linear correlation to the concentration of MsfGFP-PfODH (Figure S2A). Subsequently, the catalytic activity of the MsfGFP-PfODH samples with various concentrations were determined by measuring their respective red fluorescence $(\mathrm{Ex} / \mathrm{Em}=488 / 525 \mathrm{~nm})$ after adding coupled enzyme assay reagents. When $(R$ )-2-octanol was used as the substrate, the samples with higher concentrations of MsfGFP-PfODH exhibited higher red fluorescence intensities (Figure 5). Apparently, without considering the different amounts of enzyme in the assay, the specific activity of these enzyme samples, which was supposed to be the same, can be misrepresented based on the red fluorescence measurement. To address this limitation, we used the green fluorescence intensity, which reflected the total amount of the ADH in the assay, to normalize the red fluorescence intensity of each sample. The normalized signal of each sample was very close to each other (Figure 5), indicating similar specific activity. Moreover, Figure S3 showed that the same normalizing effect of MsfGFP's fluorescence can also be applied when a different substrate $((R)$-2-butanol) was used in the assay reaction. In addition, our further experiments also proved that this normalizing feature of MsfGFP also worked for another ADH (Figure S4). These results demonstrated that the apparent activity of ADHs can be normalized by the fluorescence of MsfGFP fusion proteins to enable a fairer comparison of enzyme mutants, thus reducing the false positive and false negative rates during screening.

\section{Using the secretion-based dual fluorescent assay in protein directed evolution}

Considering the aforementioned advantages, we combined the resorufin-based cascade reaction system and the MsfGFP-guided secretion system to establish the secretion-based dual fluorescent assay (SDFA) for directed evolution of ADHs. On the one hand, the cascade reaction system offers a sensitive and continuous determination of ADHs activity through a red fluorescence measurement. On the other hand, MsfGFP-guided secretion system provides an easy and efficient way to translocate the target $\mathrm{ADHs}$ to a simpler reaction matrix and to normalize the enzyme's activity through a green fluorescence measurement.

In the workflow of SDFA (Figure 6), the mutated genes of an ADH were first fused to the C-terminus of $m s f g f p$ to create a plasmid library. The host cells $(E$. coli $)$ were then transformed with the constructed plasmids and were spread on selection agar plates. Fully grown mutant colonies were inoculated in liquid culture medium and induced for enzyme expression. Subsequently, the cell cultures were centrifuged down to obtain the supernatants that contained the secreted enzymes for further enzyme screening. The green fluorescence of the supernatants from the enzyme mutants was first determined by a plate reader. Afterwards, assay reagents were added to the supernatant samples to monitor the red fluorescence. Finally, the catalytic activity of each mutant was normalized (red/green fluorescence) to identify the true positive mutant hits.

As a case study for demonstrating the applicability of SDFA in high-throughput screening, we employed $\mathrm{PfODH}$ as the model enzyme and sought to engineer its stereoselectivity. In oil industry, alcohol oxidation is one of the most extensively studied reactions as it plays a key role in converting petroleum-based feedstock into essential building blocks for a wide variety of chemicals (Parmeggiani, Matassini, \& Cardona, 2017). PfODH belongs to the short chain dehydrogenase/reductase (SDR) superfamily. It has a very broad substrate specificity and can catalyse the oxidation of alcohols to the corresponding aldehydes or ketones, which are the ubiquitous chemical precursors in pharmaceutical and fine chemical industries (H. Yamamoto \& Kudoh, 2013). However, PfODH has a substrate preference toward $R$-enantiomer of alcohols, thus resulting in incomplete conversion of racemic alcohol, i.e. $S$-enantiomer of alcohols cannot be fully utilized (H. Yamamoto $\&$ Kudoh, 2013). From an atom economy point of view, enzymes that can oxidize both enantiomers of alcohol are more desirable for this application.

Therefore, we set out to engineer the stereoselectivity of PfODH through screening enzyme variants with enhanced activity toward $S$-enantiomer of alcohol. To achieve this goal, we constructed a combinatorial library, PfODH-G99VWA-H150WTA-Y193RBT (containing 60 genotypes), on critical sites G99, H150, and Y193 (Figure 7), which are involved in the formation of the active cavity and extend their side chains into the cavity of PfODH. These corresponding sites were suggested as the switch to tune the stereoselectivity 
of the enzymes in the superfamily of SDRs (Qin et al., 2018). Variation of steric hindrance and polarity on sites of G99, H150, and Y193 might influence the stereo-preference of PfODH (Qin et al., 2018). Therefore, the mentioned library was constructed as the demonstration to test the applicability of SDFA as a high throughput screening method in directed evolution of ADHs.

To ensure full coverage of the design space for our constructed library, 307 clones were picked for enzyme expression. These 307 mutants were assayed by SDFA in 96 well plate format (Figure S5). Through screening, top 10 variants that exhibited the highest catalytic activity against $(S)$-2-octanol were selected for sequencing to identify their genome type. Sequence analysis revealed that 8 out of the 10 selected mutants

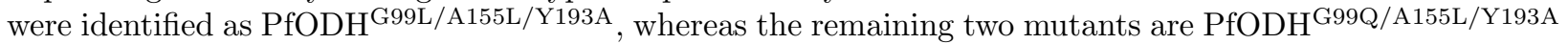
and PfODH ${ }^{\mathrm{G} 99 V / A 155 L / Y 193 A}$. Given the fact that PfODH ${ }^{\mathrm{G} 99 \mathrm{~L} / \mathrm{A} 155 \mathrm{~L} / \mathrm{Y} 193 \mathrm{~A}}$ was dominant in the top 10 positive hits and also showed higher catalytic activity than the other two variants, PfODH ${ }^{\mathrm{G} 99 V / A 155 L / Y 193 A}$ was identified as the best mutant from this library screening. To cross-validate the screening result of SDFA, PfODH ${ }^{\text {G99L/A155L/Y193A }}$ was expressed in shaking flask cultures and purified for further determining its kinetic parameter. Our results showed that $\mathrm{PfODH}$ G99L/A155L/Y193A had a 197-fold higher $\mathrm{k}_{\text {cat }} / \mathrm{k}_{\mathrm{m}}$ value toward $(S$ )-2-octanol in comparison with wild-type PfODH. These results demonstrated the capacity of SDFA to identify improved enzyme variants from a library of mutants, illustrating the usefulness of this assay in high-throughput screening of $\mathrm{ADH}$ mutants.

\section{Conclusion}

In summary, we report here the development of a sensitive, simple, and efficient fluorogenic assay that is suitable for high-throughput screening of ADHs. We demonstrated the potential of SDFA by performing directed evolution on PfODH through screening a combinatorial library. The selected enzyme variant exhibited a substantially higher $\mathrm{k}_{\text {cat }} / \mathrm{k}_{\mathrm{m}}$ value toward $(S$ )-2-octanol compared to its wild-type. In addition, the secretion-based nature of SDFA exempts the need for cell lysis, thus bypassing its disadvantages including the release of interfering cytosolic components, the additional cost of lysis reagent, the potential inconsistency of lysis efficiency, the extra effort of preserving intact culture for genome recovery. From this perspective, SDFA not only provides a sensitive and reliable way for determining ADHs activity, but also simplifies the screening procedure.

To the best of our knowledge, SDFA is the first secretion-based assay that can easily enable normalizing the catalytic activity of ADHs for high throughput screening. SDFA is in theory also applicable to other $\operatorname{NAD}(\mathrm{P})(\mathrm{H})$-dependent enzymes besides $\mathrm{ADHs}$ and is suitable for high-throughput screening methods such as $96 / 384$ well plate format. Although the method we have developed so far only works with the oxidative direction of the ADH-catalyzed reaction, SDFA should still be attractive to many protein engineers, because there are a large number of useful oxidative reactions catalyzed ADHs.

\section{Reference}

Chen, X., Liu, Z.-Q., Lin, C.-P., \& Zheng, Y.-G. (2016). Chemoenzymatic synthesis of (S)-duloxetine using carbonyl reductase from Rhodosporidium toruloides. Bioorganic Chemistry , 65 , 82-89. https://doi.org/https://doi.org/10.1016/j.bioorg.2016.02.002

Contreras-Llano, L. E., \& Tan, C. (2018). High-throughput screening of biomolecules using cell-free gene expression systems. Synthetic Biology . https://doi.org/10.1093/synbio/ysy012

Fibla, J., \& Gonzàlez-Duarte, R. (1993). Colorimetric assay to determine alcohol dehydrogenase activity. Journal of Biochemical and Biophysical Methods . https://doi.org/10.1016/0165-022X(93)90025-J

Forrest, G. L., \& Gonzalez, B. (2000). Carbonyl reductase.Chemico-Biological Interactions . https://doi.org/10.1016/S0009-2797(00)00196-4 
Fox, R. J., Davis, S. C., Mundorff, E. C., Newman, L. M., Gavrilovic, V., Ma, S. K., .. Huisman, G. W. (2007). Improving catalytic function by ProSAR-driven enzyme evolution. Nature Biotechnology . https://doi.org/10.1038/nbt1286

Held, P. (2007). Determination of NADH Concentrations with the Synergy ${ }^{\text {TM }} 2$ Multi-Detection Microplate Reader using Fluorescence or Absorbance. BioTek .

Huang, R., Chen, H., Zhong, C., Kim, J. E., \& Zhang, Y. H. P. (2016). High-Throughput Screening of Coenzyme Preference Change of Thermophilic 6-Phosphogluconate Dehydrogenase from NADP + to NAD+. Scientific Reports . https://doi.org/10.1038/srep32644

Kara, S., Spickermann, D., Weckbecker, A., Leggewie, C., Arends, I. W. C. E., \& Hollmann, F. (2014). Bioreductions catalyzed by an alcohol dehydrogenase in non-aqueous media. ChemCatChem . https://doi.org/10.1002/cctc.201300841

Ma, X., Liang, H., Cui, X., Liu, Y., Lu, H., Ning, W., ... Zhou, K. (2019). A standard for near-scarless plasmid construction using reusable DNA parts. Nature Communications . https://doi.org/10.1038/s41467019-11263-0

Makino, Y., Dairi, T., \& Itoh, N. (2007). Engineering the phenylacetaldehyde reductase mutant for improved substrate conversion in the presence of concentrated 2-propanol. Applied Microbiology and Biotechnology, 77 (4), 833-843. https://doi.org/10.1007/s00253-007-1223-2

Mayer, K. M., \& Arnold, F. H. (2002). A colorimetric Assay to Quantify Dehydrogenase Activity in Crude Cell Lysates. Journal of Biomolecular Screening . https://doi.org/10.1089/10870570252906594

Nealon, C. M., Musa, M. M., Patel, J. M., \& Phillips, R. S. (2015). Controlling Substrate Specificity and Stereospecificity of Alcohol Dehydrogenases. ACS Catalysis . https://doi.org/10.1021/cs501457v

Packer, M. S., \& Liu, D. R. (2015). Methods for the directed evolution of proteins. Nature Reviews Genetics . https://doi.org/10.1038/nrg3927

Parmeggiani, C., Matassini, C., \& Cardona, F. (2017). A step forward towards sustainable aerobic alcohol oxidation: New and revised catalysts based on transition metals on solid supports. Green Chemistry . https://doi.org/10.1039/c7gc00406k

Pédelacq, J. D., Cabantous, S., Tran, T., Terwilliger, T. C., \& Waldo, G. S. (2006). Engineering and characterization of a superfolder green fluorescent protein. Nature Biotechnology . https://doi.org/10.1038/nbt1172

Qin, F., Qin, B., Zhang, W., Liu, Y., Su, X., Zhu, T., ... You, S. (2018). Discovery of a Switch Between Prelog and Anti-Prelog Reduction toward Halogen-Substituted Acetophenones in Short-Chain Dehydrogenase/Reductases. ACS Catalysis , 8 (7), 6012-6020. https://doi.org/10.1021/acscatal.8b00807

Savile, C., Gruber, J. M., Mundorff, E., Huisman, G., \& Collier, S. J. (2014). Ketoreductase polypeptides for the production of a 3-aryl-3-hydroxypropanamine from a 3-aryl-3-ketopropanamine . Retrieved from https://www.google.ch/patents/US20100151534

Simeonov, A., \& Davis, M. I. (2004). Interference with Fluorescence and Absorbance. In Assay Guidance Manual .

Tjalsma, H., Bolhuis, A., Jongbloed, J. D. H., Bron, S., \& van Dijl, J. M. (2000). Signal Peptide-Dependent Protein Transport in Bacillus subtilis: a Genome-Based Survey of the Secretome. Microbiology and Molecular Biology Reviews . https://doi.org/10.1128/mmbr.64.3.515-547.2000

Yamamoto, H., \& Kudoh, M. (2013). Novel chiral tool, (R)-2-octanol dehydrogenase, from Pichia finlandica: purification, gene cloning, and application for optically active $\alpha$-haloalcohols. Applied Microbiology and Biotechnology , 97 (18), 8087-8096. https://doi.org/10.1007/s00253-012-4643-6 
Yamamoto, K., Kurisu, G., Kusunoki, M., Tabata, S., Urabe, I., \& Osaki, S. (2001). Crystal structure of glucose dehydrogenase from Bacillus megaterium IWG3 at $1.7 \AA$ resolution. Journal of Biochemistry . https://doi.org/10.1093/oxfordjournals.jbchem.a002858

Zeymer, C., \& Hilvert, D. (2018). Directed Evolution of Protein Catalysts. Annual Review of Biochemistry . https://doi.org/10.1146/annurev-biochem-062917-012034

Zhang, H., Lountos, G. T., Ching, C. B., \& Jiang, R. (2010). Engineering of glycerol dehydrogenase for improved activity towards 1, 3-butanediol. Applied Microbiology and Biotechnology . https://doi.org/10.1007/s00253-010-2735-8

Zhang, R., Xu, Y., \& Xiao, R. (2015). Redesigning alcohol dehydrogenases/reductases for more efficient biosynthesis of enantiopure isomers. Biotechnology Advances . https://doi.org/10.1016/j.biotechadv.2015.08.002

Zhang, Z., Tang, R., Zhu, D., Wang, W., Yi, L., \& Ma, L. (2017). Non-peptide guided auto-secretion of recombinant proteins by super-folder green fluorescent protein in Escherichia coli.Scientific Reports . https://doi.org/10.1038/s41598-017-07421-3

Zheng, Y. G., Yin, H. H., Yu, D. F., Chen, X., Tang, X. L., Zhang, X. J., .. Liu, Z. Q. (2017). Recent advances in biotechnological applications of alcohol dehydrogenases. Applied Microbiology and Biotechnology . https://doi.org/10.1007/s00253-016-8083-6

\section{Figure legend:}

Figure 1 Working principle of SDFA. Target ADH is translocated to the extracellular environment by a MsfGFP-guided secretion system. Subsequently, the enzyme activity of ADH is determined by a cascade reaction in which $\mathrm{NADH}$ produced by the $\mathrm{ADH}$-catalyzed reaction was used by diaphorase to reduce resazurin into resorufin, which is highly fluorescent. When excessive amount of resazurin and diaphorase were provided, the rate of NADH formation was proportional to the rate at which the red fluorescence increased.

Figure 2. Catalytic activity of PfODH toward various substrates determined by the coupled enzyme assay. The increase of resorufin fluorescence $(\mathrm{Ex} / \mathrm{Em}=535 / 588 \mathrm{~nm})$ was monitored for the PfODH-catalyzed reactions with (A) ( $R$ )-2-octanol, (B)1-(3-Methylphenyl)ethanol, (C) 4-Fluoro- $\alpha$-methylbenzyl alcohol, and (D) 1-Phenylethanol respectively. All assays were performed in a final reaction volume of $100 \mu \mathrm{l}$ with the following conditions: $10 \mu \mathrm{g} / \mathrm{ml}$ purified PfODH, $2 \mathrm{U} / \mathrm{ml}$ diaphorase, $0.5 \mathrm{mM}$ resazurin, $0.5 \mathrm{mM}$ alcoholic substrate, $0.5 \mathrm{mM} \mathrm{NAD}{ }^{+}, 0.1 \mathrm{M}$ Tris solution ( $\mathrm{pH}$ 8.0). Assay reactions without adding purified PfODH served as the controls for each substrate reaction. RFU: relative fluorescence units.

Figure 3 . Fluorescence image of the supernatants of the cells expressing the MsfGFP/eGFP fusion proteins or eGFP (upper part of the figure). The supernatant samples were excited by blue light (Safe Imager 2.0 Blue Light Transilluminator, Thermo) and the image was taken through an amber filter unit. SDS-PAGE analysis of the concentrated supernatant samples of the cells expressing the MsfGFP/eGFP fusion proteins or eGFP (lower part of the figure).

Figure 4. Catalytic activity of the secreted MsfGFP fusion proteins determined by the coupled enzyme assay. The increase of resorufin fluorescence $(\mathrm{Ex} / \mathrm{Em}=535 / 588 \mathrm{~nm})$ was monitored for the reactions catalyzed by the secreted MsfGFP fusion proteins. (A)MsfGFP-GlyDH and eGFP-GlyDH were characterized by using 1,3-butanediol as the substrate. (B) MsfGFP-CpSADH and eGFP-CpSADH were characterized by using $(S$ )-2-octanol as the substrate.(C) MsfGFP-PfODH and eGFP-PfODH were characterized by using $(R$ )-2-octanol as the substrate. (D) MsfGFP-BDH_KP and eGFP-BDH_KP were characterized by using Meso2,3-butanediol as the substrate. The assay was carried out by mixing the supernatant sample and the assay reagent mixture at volumetric ratio of 1:1. All assays were performed in a final reaction volume of $100 \mu \mathrm{l}$ 
with the following conditions: $50 \mu \mathrm{l}$ supernatant sample, $2 \mathrm{U} / \mathrm{ml}$ diaphorase, $0.5 \mathrm{mM}$ resazurin, $0.5 \mathrm{mM}$ alcoholic substrate, $0.5 \mathrm{mM} \mathrm{NAD}^{+}, 0.05 \mathrm{M}$ Tris solution ( $\mathrm{pH}$ 8.0). RFU: relative fluorescence units.

Figure 5. Catalytic activity of ADHs normalized by the fluorescence of MsfGFP. Reactions catalyzed by various concentrations of MsfGFP-PfODH with substrate $(R)$-2-octanol. Red fluorescence represents the fluorescence intensity of resorufin $(\mathrm{Ex} / \mathrm{Em}=535 / 588 \mathrm{~nm})$ recorded at a time point within the linear part of the progress curve of MsfGFP-PfODH catalyzed reactions. The same time point measurement was taken for all the reactions with various enzyme concentrations. Green fluorescence represents the fluorescence intensity of MsfGFP-PfODH. The normalized signal is calculated by dividing the red fluorescence signal by the green fluorescence signal. The assay was carried out by mixing the MsfGFP-PfODH sample and the assay reagent mixture at volumetric ratio of 1:1. All assays were performed in a final reaction volume of $100 \mu \mathrm{l}$ with the following conditions: $50 \mu \mathrm{l}$ enzyme sample, $2 \mathrm{U} / \mathrm{ml}$ diaphorase, $0.5 \mathrm{mM}$ resazurin, $0.5 \mathrm{mM}$ alcoholic substrate, $0.5 \mathrm{mM} \mathrm{NAD}^{+}, 0.05 \mathrm{M}$ Tris solution ( $\mathrm{pH} 8.0$ ). Each bar indicates the mean value of three biological replicates and the corresponding error bar indicates the standard deviation. RFU: relative fluorescence units.

Figure 6. Schematic overview of the SDFA workflow for the directed evolution of ADHs. 1-2: Mutated ADHs' genes were fused to the C-terminus of MsfGFP to create a plasmid library. 3-4: The host cells were transformed with the constructed plasmids and were spread on selection agar plates. 5: Grown mutant colonies were inoculated in liquid medium and induced for enzyme expression. 6: The cell cultures were centrifuged down to obtain the supernatants that contained the secreted enzymes. The green fluorescence of the supernatants from the enzyme mutants was determined. 7: Assay reagents were added to the supernatant samples to monitor the red fluorescence. 8: The catalytic activity of each mutant was normalized to identify positive hits.

Figure 7. The predicted conformations of (R)-2-octanol (cyan ball-stick) and $\mathrm{NAD}^{+}$(yellow stick) in the active site of PfODH. The catalytic residues (S148 and Y161) are shown in blue sticks. The critical residues (G99, H150, and Y193), which affect the substrate specificity and enantioselectivity, are shown in green sticks. The substrate, $(R)$-2-octanol, was built by the builder tool of PyMol (DeLano Scientific) in productive conformation. Model structure of PfODH-NAD ${ }^{+}$complex was constructed based on homology modeling method using Swiss-Model server (https://swissmodel.expasy.org). The structure of glucose dehydrogenase from Bacillus megaterium IWG3 (K. Yamamoto et al., 2001), BmGDH (PDB: 1GCO), was selected as the template. They share $40 \%$ sequence identity.

\section{Hosted file}

Figures.pdf available at https://authorea.com/users/354725/articles/478195-a-secretionbased-dual-fluorescence-assay-for-high-throughput-screening-of-alcohol-dehydrogenases 